\section{Programa piloto para la gestión energética en instituciones educativas del departamento del Tolima}

\author{
Program for energy management in the educational \\ institutions of the department of Tolima
}

Recibido: 15-feb-17 - Revisado: 30-abr-17

Aceptado: 30-may-17 - Publicado: 30-jul-17

\section{Giovanni Andrés Vargas Galván}

Director de Programa, Facultad de Ciencias Administrativas y Contables, Tecnologia en Gestión Ambiental,
Grupo Virtual Tec Corporación de Educación del Norte Grupo Virtual Tec, Corporacion de Educacion barrio El Reposo, Honda, Colombia. gestionambiental@coreducacion.edu.co

\section{Agustín Valverde Granja}

Docente de tiempo completo, Facultad de Ingenierías, Programa de Ingeniería Mecánica, Universidad de lbagué, Carrera 22 Calle 67 Barrio Ambalá, Ibagué, Colombia. agustin.valverde@unibague.edu.co

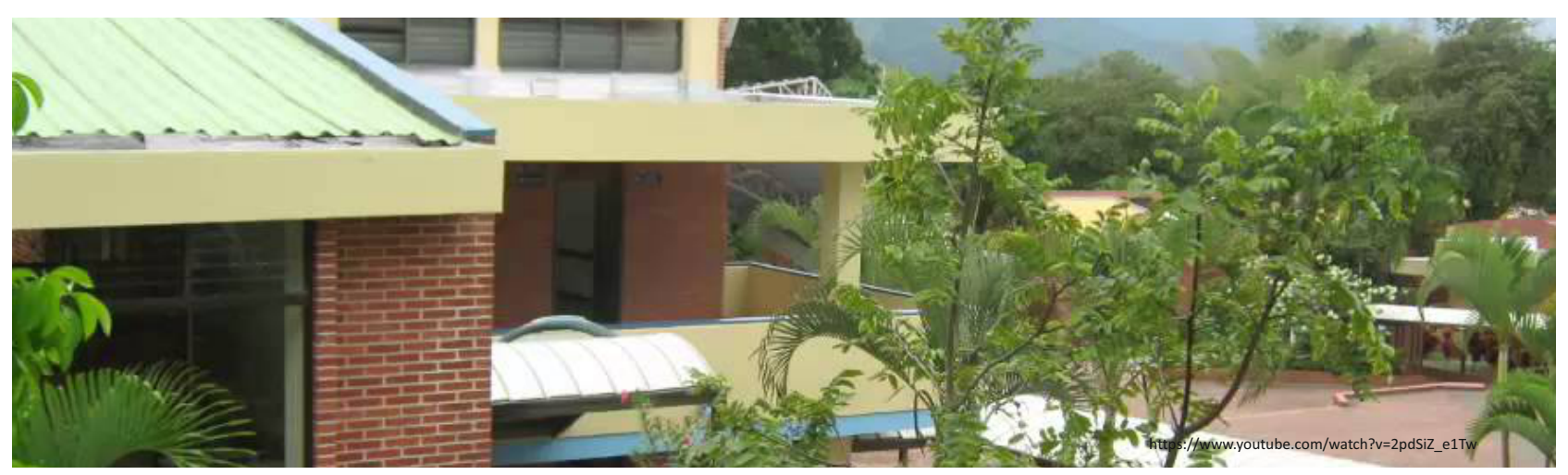

Resumen: En este artículo se presenta el desarrollo de un programa piloto para la gestión energética que permita mejorar el desempeño de factores financieros, ambientales, sociales, operacionales y organizacionales de las instituciones educativas en el departamento del Tolima, tomando como caso de estudio el Colegio Champagnat de Ibagué. Dentro de los resultados obtenidos, se tienen: la caracterización energética del Colegio; la definición de indicadores de gestión, donde se obtuvo: índice de consumo energético de 1,01 kWh/mes- $\mathrm{m}^{2}$, índice de potencia instalada por área total de 16,18 W/m², índice de consumo energético de $8,18 \mathrm{kWh} / \mathrm{mes}$-persona, índice de potencia instalada de 130,96 W/persona, huella de carbono de $2.100 \mathrm{~kg} \mathrm{CO} 2 \mathrm{e} / \mathrm{mes}$, índice de emisiones por área de edificio de 0,19 kg CO2/mes-m², índice de emisiones de 1,54 kg $\mathrm{CO}_{2}$ /mes-persona; la capacitación de 105 personas entre administrativos, docentes y estudiantes en gestión de la energía; y una propuesta de un programa piloto para los colegios oficiales en el departamento del Tolima. Adicional a lo anterior, se elaboraron tres (propuestas a corto plazo, una a mediano plazo y dos a largo plazo; así, por mes, se estimó un ahorro energético del 38,19\% y por año, una reducción en la huella de carbono de doce toneladas de $\mathrm{CO}_{2} \mathrm{e}$.

Palabras clave: Energía activa, gestión energética, indicadores de gestión, ahorro energético.

Abstract: project aims to develop a pilot program for energy management that allows to enhance the performance of finantial, enviromental, social, operational and organizational factors of educational institutions in the Tolima State, taking as case study the school Champañat in Ibagué city. The collected results, are: the energy characterization of the school; the definition of the management indicators such as: energy consumption rate of $1,01 \mathrm{kWh} / \mathrm{month}$ - $\mathrm{m}^{2}$, installed power rate per total area of $16,18 \mathrm{~W} / \mathrm{m}^{2}$, energy consumption rate of $8,18 \mathrm{kWh} / \mathrm{month}$-person, installed power rate of $130,96 \mathrm{~W} / \mathrm{person}$, carbon footprint of $2.100 \mathrm{~kg} \mathrm{CO} 2 \mathrm{e} /$ month, emission rate per building area of $0,19 \mathrm{~kg} \mathrm{CO} / \mathrm{month}^{2} \mathrm{e}$, emission rate of 1,54 kg CO 2 /month-person.; the training of 105 people among administrative assistants, professors and students in energy management and a proposal of a pilot program for the schools in Tolima department; additionally, three short term proposals were developed, one (1) in the medium term and two (2) in the long one; estimating an energy saving of 38,19\% monthly and a carbon footprint reduction of 12 tons of $\mathrm{CO}_{2} \mathrm{e}$ annually.

Key words: Active energy; energy management; management indicators; energy saving. 


\section{INTRODUCCIÓN}

La energía se ha convertido en uno de los aspectos clave que las empresas productivas y de servicios deben controlar y mejorar en su gestión. Uno de los mecanismos utilizados para este fin es la implementación de sistemas de gestión energética que traen consigo la reducción de los costos financieros por consumos energéticos (Bunse et al., 2011), la mejora de la imagen corporativa (Dobes, 2013) y la reducción del impacto ambiental (Skip Laitner, 2013). Por lo tanto, la energía se está convirtiendo en un factor de producción cada vez más crítico y su gestión adecuada puede dar a las empresas una ventaja competitiva importante $y$ estratégica de activos, lo que les permite reaccionar a los cambios de escenarios de desarrollo flexible; así, con una profunda adopción de prácticas de gestión de la energía, puede considerarse un indicador efectivo de la rentabilidad futura (Skip Laitner, 2013).

A pesar de ello, existen empresas donde la gestión de la energía todavía tiene baja prioridad y los intentos se realizan de forma aislada para reducir consumos energéticos en lugar de un enfoque sistémico para resolver el problema (Introna et al., 2014).

La metodología implementada en este proyecto fue la Gestión Total Eficiente de la Energía (Campos Avella et al., 1997), que se adapta a las necesidades de las instituciones educativas y también a la norma ISO 50001:2011 de la auditoría energética (Organización Internacional de Normalización, 2011); además, esta ha tenido un uso generalizado en alrededor de un millar de entidades públicas y privadas en varias naciones de Latinoamérica, dentro de ellas, Colombia. Por otro lado, las normas que auditan e incentivan el uso racional y eficiente de la energía para empresas productivas y de servicios en Colombia son la Ley 697 de 2001 (Gobierno Nacional, 2001), el Decreto 3683 de 2003 (Gobierno Nacional, 2003), el Decreto 2331 de 2007 y las Normas ISO 50000 (Unidad de Planeación Minero Energética, 2013).

\section{MATERIALES Y MÉTODOS}

La metodología utilizada fue la Gestión Total Eficiente de la Energía (Campos Avella et al., 1997), la cual consta de las siguientes fases:

\subsection{Caracterización energética del colegio Champagnat de lbagué}

La caracterización energética realizada al colegio Champagnat de la ciudad de Ibagué se muestra a continuación.

\subsubsection{Diagrama de proceso energético de la institución educativa}

La Fig. 1 representa el diagrama energético con datos correspondientes a las visitas de campo realizadas al colegio durante el año 2014. Como se observa, el consumo eléctrico es inherente a todas las áreas, incluso a aquellas indirectamente relacionadas con el proceso educativo, como la iluminación pública.

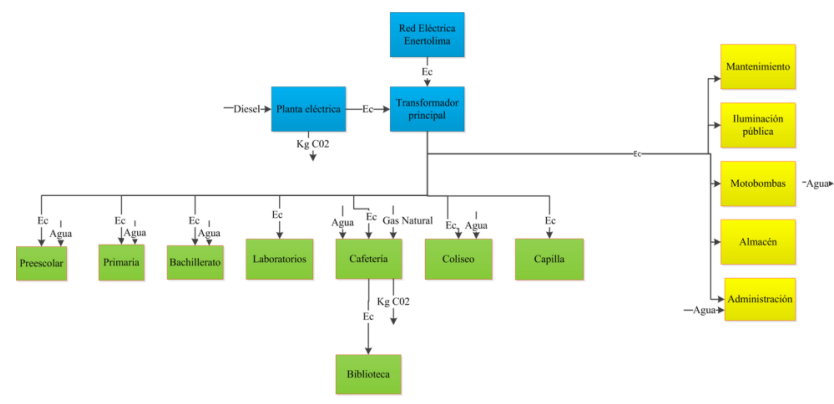

Fig. 1. Diagrama energético del colegio Champagnat.

\subsubsection{Consumo energético por hora}

Para realizar la toma de datos del consumo de energía activa ( $k W h$ ) en el colegio Champagnat por hora, se utilizó el analizador de potencia marca Hioki 3360-21. Este fue instalado de acuerdo a la norma de calidad de energía NTC 5001:2008 (Norma Técnica Colombiana, 2008) en el breaker principal y en los secundarios que identificaban las áreas de la institución. 


\subsubsection{Comportamiento del consumo de energía} eléctrica en el Colegio Champagnat

En este estudio, se realizaron diversos modelos con el fin de determinar la línea base para una posible proyección del consumo energético en instituciones educativas; sin embargo, no se logró determinar una ecuación para dicho fin, debido a la alta variación de los consumos energéticos; mientras que la cantidad de personal, equipos y los metros cuadrados por áreas se mantiene casi constante.

\subsubsection{Diagrama de Pareto para las áreas del Colegio Champagnat}

El diagrama de Pareto se realizó con base en los datos de consumo del analizador de potencia Hioki 3360-21 para el colegio Champagnat por mes y se muestra en la Fig. 2; además, en la misma figura, se muestra que las áreas críticas de consumo energético en kWh por mes son: motobombas, con 2410; seguido de bachillerato, con 1695; biblioteca-cafetería, 1628; laboratorios, 1194 y el coliseo, con 894. De igual forma, el procedimiento se realizó con cada una de las áreas críticas para determinar los equipos de mayor consumo energético en la institución.

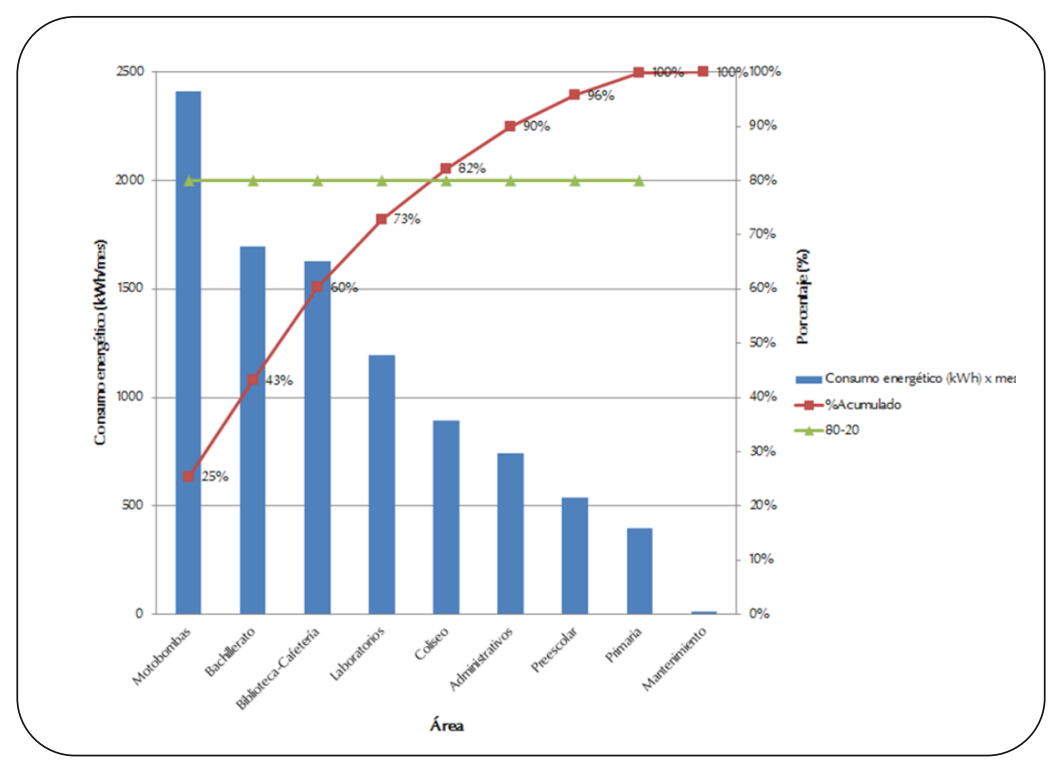

Fig. 2. Diagrama de Pareto áreas colegio Champagnat.

2.2 Definición y validación de indicadores de gestión enfocados al ahorro energético y sostenibilidad

\subsubsection{Propuesta de indicadores de gestión para instituciones educativas de nivel secundaria} Se proponen los siguientes indicadores de gestión, de acuerdo a cuatro niveles que se mencionan a continuación: (a) Nivel 1: Indicadores por dependencias/zonas u áreas/pisos (Pinzón C. et al., 2014). En la Tabla 1, se muestran los indicadores propuestos de nivel 1, la unidad y la fuente de información para realizar el seguimiento.

(b) Nivel 2: Indicadores para equipos/sistemas principales (Pinzón C. et al., 2014). En la Tabla 2 , se muestran los indicadores propuestos de nivel 2, la unidad y la fuente de información para realizar el seguimiento. 
Nivel 3: Indicadores por el establecimiento (Pinzón C. et al., 2014). En la Tabla 3, se evidencian los indicadores propuestos de nivel 3, la unidad y la fuente de información para realizar el seguimiento. (d) Nivel 4: Indicadores de emisiones de CO2. En la Tabla 4, se muestran los indicadores propuestos de nivel 4, la unidad y la fuente de información para realizar el seguimiento.

Tabla 1. Nivel 1 de indicadores de gestión en instituciones educativas.

\begin{tabular}{|c|c|c|c|}
\hline Nivel & Indicador & Unidad & Información \\
\hline \multirow{2}{*}{$\begin{array}{l}\text { 1: Indicadores } \\
\text { por } \\
\text { dependencias } \\
\text { /zonas u } \\
\text { áreas/pisos }\end{array}$} & $\begin{array}{l}\text { Índice de } \\
\text { consumo } \\
\text { energético por } \\
\text { área }\end{array}$ & $\mathrm{kWh} / \mathrm{mes}-\mathrm{m}^{2}$ & $\begin{array}{l}\text { Los consumos a partir de los medidores (contadores } \\
\text { eléctricos o equipos) y las dimensiones físicas } \\
\text { construidas (en } \mathrm{m}^{2} \text { ) de los planos arquitectónicos. }\end{array}$ \\
\hline & $\begin{array}{l}\text { Índice de potencia } \\
\text { instalada por área }\end{array}$ & $\mathrm{W} / \mathrm{m}^{2}$ & $\begin{array}{l}\text { Potencia instalada del censo de carga o inventario de } \\
\text { equipos actualizado y el área de los planos } \\
\text { arquitectónicos. }\end{array}$ \\
\hline
\end{tabular}

Fuente: Pinzón C. et al., 2014.

Tabla 2. Nivel 2 de indicadores de gestión en instituciones educativas.

\begin{tabular}{|c|l|c|l|}
\hline Nivel & \multicolumn{1}{|c|}{ Indicador } & Unidad & \multicolumn{1}{c|}{ Información } \\
\hline $\begin{array}{c}\text { 2: Indicadores } \\
\text { para } \\
\text { equipos/sistemas } \\
\text { principales }\end{array}$ & $\begin{array}{l}\text { Índice de consumo energético } \\
\text { por aplicación }\end{array}$ & $\mathrm{kWh} / \mathrm{mes}-\mathrm{m}^{2}$ & $\begin{array}{l}\text { Los consumos a partir de los medidores (contadores } \\
\text { eléctricos o equipos) a instalar y las dimensiones } \\
\text { físicas construidas (en } \mathrm{m}^{2} \text { ) de los planos } \\
\text { arquitectónicos. }\end{array}$ \\
\cline { 2 - 4 } & $\begin{array}{l}\text { Índice de potencia instalada por } \\
\text { aplicación }\end{array}$ & $\mathrm{W} / \mathrm{m}^{2}$ & $\begin{array}{l}\text { Potencia instalada del aforo de carga o inventario de } \\
\text { equipos actualizado y el área de los planos } \\
\text { arquitectónicos. }\end{array}$ \\
\hline
\end{tabular}

Fuente: Pinzón C. et al., 2014.

Tabla 3. Nivel 3 de indicadores de gestión en instituciones educativas.

\begin{tabular}{|c|c|c|c|}
\hline Nivel & Indicador & Unidad & Información \\
\hline \multirow{4}{*}{$\begin{array}{l}\text { 3: Indicadores } \\
\text { por el } \\
\text { establecimiento }\end{array}$} & $\begin{array}{l}\text { Índice de consumo energético } \\
\text { por área total. }\end{array}$ & $\mathrm{kWh} / \mathrm{mes}-\mathrm{m}^{2}$ & $\begin{array}{l}\text { A partir de las facturas de energía y de los } \\
\text { planos arquitectónicos. }\end{array}$ \\
\hline & $\begin{array}{l}\text { Índice de potencia instalada } \\
\text { por área total. }\end{array}$ & $\mathrm{W} / \mathrm{m}^{2}$ & $\begin{array}{l}\text { Potencia total instalada del inventario de } \\
\text { equipos o de un ce nso de carga y área de los } \\
\text { planos arquitectónicos. }\end{array}$ \\
\hline & $\begin{array}{l}\text { Índice de consumo energético } \\
\text { por persona (Per Cápita). }\end{array}$ & kWh / mes-persona & $\begin{array}{l}\text { Número total de administrativos, docentes y } \\
\text { estudiantes. }\end{array}$ \\
\hline & $\begin{array}{l}\text { Índice de potencia instalada } \\
\text { por persona. }\end{array}$ & W / persona & $\begin{array}{l}\text { Potencia total instalada del inventario de } \\
\text { equipos o censo de carga y con el número total } \\
\text { de empleados sumado a los estudiantes. }\end{array}$ \\
\hline
\end{tabular}

Fuente: Pinzón C. et al., 2014. 
Tabla 4. Nivel 4 de indicadores de gestión en instituciones educativas.

\begin{tabular}{|c|c|c|c|}
\hline Nivel & Indicador & Unidad & Información \\
\hline \multirow{3}{*}{$\begin{array}{l}\text { 4: Indicadores } \\
\text { de emisiones }\end{array}$} & $\begin{array}{l}\text { Huella de carbono (Kg CO } 2 \\
\text { equivalentes)-Alcances 1,2 } \\
\text { y/o 3. Norma ISO 14064-1 o } \\
\text { el método de las cuentas } \\
\text { contable (MC3). }\end{array}$ & $\mathrm{KgCO}_{2} \mathrm{e} /$ mes & $\begin{array}{l}\text { Convertir los consumos energéticos en emisiones } \\
\text { de } \mathrm{CO}_{2} \text { con los índices actuales Mix eléctrico y } \\
\text { combustibles fósiles. }\end{array}$ \\
\hline & $\begin{array}{l}\text { Índice de emisiones de } \mathrm{CO}_{2} \\
\text { por área de edificio }\end{array}$ & $\begin{array}{l}\mathrm{kgCO}_{2} / \text { mes-m² } \\
\mathrm{kgCO}_{2} / \text { año-m² }\end{array}$ & $\begin{array}{l}\text { Convertir los consumos energéticos en emisiones } \\
\text { de } \mathrm{CO}_{2} \text { con el índice actual de Mix eléctrico. }\end{array}$ \\
\hline & $\begin{array}{l}\text { Índice de emisiones de } \mathrm{CO}_{2} \\
\text { por persona (Per Cápita) }\end{array}$ & $\begin{array}{l}\mathrm{kgCO}_{2} / \text { mes-persona } \\
\mathrm{kgCO}_{2} / \text { año-persona }\end{array}$ & $\begin{array}{l}\text { Convertir los consumos energéticos en emisiones } \\
\text { de } \mathrm{CO}_{2} \text { con el índice actual de Mix eléctrico y con } \\
\text { el número total de empleados sumado a los } \\
\text { estudiantes. }\end{array}$ \\
\hline
\end{tabular}

Fuente: Pinzón C. et al., 2014.

\subsection{Formación de talento humano en el colegio Champagnat}

En el colegio Champagnat se realizó la capacitación de 20 administrativos, 32 docentes y 53 estudiantes de la institución en temas de gestión energética, que equivale al $12 \%$ del personal. Lo anterior con la finalidad de conformar el Comité de Ahorro Energético y Medio Ambiente (CAEMA) con integrantes capacitados.

\section{ANÁLISIS Y DISCUSIÓN DE RESULTADOS}

3.1 Gestión de indicadores para el Colegio Champagnat con propuestas a corto, mediano y largo plazo

En la Tabla 5, se observa el consumo energético que se espera ahorrar con la implementación de las propuestas realizadas a corto, mediano y largo plazo (iluminación led). Dicho consumo es de 3770 kWh mensuales y equivalente al 38,19 \% del consumo energético total; lo cual, cumple con los ahorros energéticos estimados entre el 30 y $42 \%$ en las instituciones educativas de primaria y secundaria (Torres E. et al., 2002; Economou, 2012; Dias Pereira et al., 2014; Arambula Lara et al., 2015; Kim \& Hong, 2012; Zapata \& González, 2014).

Además, se espera una reducción de doce toneladas de CO2e/año (46,31\%), lo que evidenciaría una oportunidad de aportar a prevenir el cambio climático debido a los gases de efecto invernadero. De igual forma, se elaboraron otras dos propuestas, que fueron: un sistema fotovoltaico con conexión a red de 335 kWh y la conformación del CAEMA.

Tabla 5. Energía activa ahorrada por propuestas a corto, mediano y largo plazo.

\begin{tabular}{|l|c|}
\hline \multicolumn{1}{|c|}{ Propuesta } & Energía activa ahorrada (kWh/mes) \\
\hline Configuración modo ahorro de energía en equipos de cómputo. & 21,28 \\
\hline $\begin{array}{l}\text { Independizar los interruptores del encendido de las luminarias para sectorizar su } \\
\text { consumo energético y establecer un horario adecuado de rondas de vigilancia y } \\
\text { aseo para evitar que los equipos queden encendidos. }\end{array}$ & 175,75 \\
\hline Cambio de motobombas de la piscina y abastecimiento de agua. & 1544,03 \\
\hline Cambio de iluminación incandescente y fluorescente a led. & 2029 \\
\hline Total & $\mathbf{3 7 7 0}$ \\
\hline
\end{tabular}


A partir de los indicadores planteados y de las oportunidades de ahorro que existen, se muestra el listado de indicadores que aplican para la comparación y las metas esperadas para ellos (ver Tabla 6). Asimismo, se presenta la comparación de los indicadores del Colegio Champagnat para el año 2014 (ver Tabla 7), con el único caso de estudio que se encontró en la literatura para Colombia, el cual fue el Edificio Educativo Alejandro Suárez Copete de la Universidad Distrital en la ciudad de Bogotá (Pinzón C. et al., 2014).
Además, se determinó que todos los indicadores tienen menor valor y sus diferencias oscilan entre el 23 y $96 \%$. Lo anterior debido principalmente a que en el edificio, el consumo energético promedio anual fue de 109540 kWh y el del colegio es de 9787 kWh; adicionalmente, el número de personas en el edificio en promedio fue de 566 personas, mientras que en el colegio, el promedio fue de 1195 personas. También, cabe anotar que el número de equipos por área geométrica es diferente.

Tabla 6. Comparación de indicadores actuales y meta, año 2014.

\begin{tabular}{|c|c|c|c|c|c|}
\hline Nivel & Indicador & Unidad & Valor actual & Valor meta & \% disminución \\
\hline 3 & $\begin{array}{l}\text { Índice de consumo energético } \\
\text { por área total. }\end{array}$ & $\mathrm{kWh} / \mathrm{mes}-\mathrm{m}^{2}$ & 1,01 & 0,62 & $39 \%$ \\
\hline 3 & $\begin{array}{l}\text { Índice de potencia instalada } \\
\text { por área total. }\end{array}$ & $\mathrm{W} / \mathrm{m}^{2}$ & 16,18 & 13,31 & $18 \%$ \\
\hline 3 & $\begin{array}{l}\text { Índice de consumo energético } \\
\text { por persona. }\end{array}$ & kWh / mes-persona & 8,18 & 5,03 & $39 \%$ \\
\hline 3 & $\begin{array}{l}\text { Índice de potencia instalada } \\
\text { por persona. }\end{array}$ & W / persona & 130,96 & 107,69 & $18 \%$ \\
\hline 4 & Huella de carbono & $\mathrm{KgCO}_{2} \mathrm{e} / \mathrm{mes}$ & 2100 & 1167 & $44 \%$ \\
\hline 4 & $\begin{array}{l}\text { Índice de emisiones de } \\
\text { por área de edificio }\end{array}$ & $\mathrm{kgCO}_{2} / \mathrm{mes}-\mathrm{m}^{2}$ & 0,19 & 0,12 & $37 \%$ \\
\hline 4 & $\begin{array}{l}\text { Índice de emisiones de } \\
\text { por persona }\end{array}$ & $\mathrm{kgCO}_{2} /$ mes-persona & 1,54 & 0,95 & $38 \%$ \\
\hline \multicolumn{5}{|c|}{ Promedio } & $34 \%$ \\
\hline
\end{tabular}

Tabla 7. Comparación de indicadores de gestión con otras instituciones educativas.

\begin{tabular}{|c|c|c|c|c|}
\hline Nivel & Indicador & Unidad & $\begin{array}{c}\text { Colegio } \\
\text { Champagnat }\end{array}$ & $\begin{array}{l}\text { Edificio Suárez } \\
\text { Copete }\end{array}$ \\
\hline 2 & $\begin{array}{l}\text { Índice de consumo energético por } \\
\text { aplicación (Iluminación) }\end{array}$ & $\mathrm{kWh} / \mathrm{mes}-\mathrm{m}^{2}$ & 2,13 & 2,76 \\
\hline 2 & $\begin{array}{l}\text { Índice de consumo energético por } \\
\text { aplicación (Equipos de cómputo). }\end{array}$ & $\mathrm{kWh} / \mathrm{mes}^{-\mathrm{m}^{2}}$ & 0,23 & 3,05 \\
\hline 3 & $\begin{array}{l}\text { Índice de consumo energético por } \\
\text { área total. }\end{array}$ & $\mathrm{kWh} / \mathrm{mes}-\mathrm{m}^{2}$ & 1,01 & 25,13 \\
\hline 3 & $\begin{array}{l}\text { Índice de consumo energético por } \\
\text { persona. }\end{array}$ & kWh / mes-persona & 8,18 & 122,05 \\
\hline 4 & $\begin{array}{l}\text { Índice de emisiones de } \quad 2 \text { por } \\
\text { área de edificio }\end{array}$ & $\mathrm{kgCO}_{2} / \mathrm{mes}-\mathrm{m}^{2}$ & 0,19 & 3,02 \\
\hline 4 & $\begin{array}{l}\text { Índice de emisiones de } 2 \text { por } \\
\text { persona }\end{array}$ & $\mathrm{kgCO}_{2} /$ mes-persona & 1,54 & 14,65 \\
\hline
\end{tabular}


Por otro lado, cuando se comparó el índice de consumo energético por área total ( $\mathrm{kWh} / \mathrm{año}-\mathrm{m} 2)$ del Champagnat, que fue de 12,12 , con los obtenidos en los colegios de secundaria a nivel internacional (Europa, Asia, Centroamérica y Suramérica), se determinó que estaba por debajo del rango promedio estándar, equivalente a 100 kWh/año-m² (Yan et al., 2011; Dias Pereira et al., 2014).

Adicionalmente, se clasificó el colegio en un rango insuficiente de eficiencia energética. Esto indica que en los colegios colombianos, aún el nivel tecnológico para algunas aplicaciones (equipos o sistemas) es bajo y que la distribución de los equipos en las áreas geométricas de las instituciones en su mayoría no es la adecuada o no se basa en estudios serios de ingeniería o arquitectura para hacerla, lo cual, permitiría tener espacios muy grandes con poco consumo energético y viceversa. No obstante, se debe tener en cuenta que para llegar a una mejor comparación, se tendría que analizar cómo el factor climático (grados-día) afecta el consumo energético en este tipo de instituciones educativas.

También, al comparar el valor del indicador Huella de Carbono del colegio Champagnat (25,3 toneladas de CO2 equivalentes para el año 2014) con otras universidades (Dávila Collaguazo \& Varela Rosario, 2014; Ozawa-Meida et al., 2013; Álvarez et al., 2014; N. Larsen et al., 2013; Townsend \& Barrett, 2013), colegios y escuelas (Valencia Toro \& Vargas Londoño, 2013), se concluye que en el caso de los colegios de secundaria y escuelas de veredas, tal indicador es mayor; mientras que en el caso de las universidades, es menor. Esto se debe a que dichas instituciones de educación superior tienen mayor infraestructura física, por ende, mayor consumo energético y mayor número de personal con respecto a los colegios de educación media.

De esta forma, se puede apreciar que todos los indicadores involucrados disminuyen con la implementación de las propuestas en un valor promedio de $34 \%$, por lo tanto, se hace necesario un seguimiento continuo de los indicadores; además, los índices propuestos permitirán realizar comparaciones no solo entre colegios de educación secundaria a nivel del departamento del Tolima y nacional, sino también internacional; esto de acuerdo a: número de equipos y su potencia nominal, cantidad de personal y el área de ocupación.

En este último, se debe tener en cuenta que para comparar los colegios, se analizan los sistemas de calefacción y refrigeración y el diseño constructivo de la infraestructura física, es decir, que aplicaría para ciudades con climas fríos, como por ejemplo, Bogotá.

\subsection{Propuesta de programa piloto de gestión energética en el departamento del Tolima}

En la Fig. 3, se muestra el programa piloto de gestión energética propuesto para realizar la implementación en las instituciones educativas en el departamento del Tolima. Este fue elaborado utilizando la técnica Diagrama de Flujo (San Miguel, 2009), con el fin de buscar establecer la dinámica de los principales componentes que aportan funcionalidad al programa. Se espera que la implementación del programa piloto de gestión energética permita realizar la mejora de factores como el financiero (costo energía), el ambiental (emisiones de $\mathrm{CO}_{2}$ ), social (se espera capacitar en gestión energética para el departamento del Tolima 327420 personas), operacional (número de actividades reformadas para lograr establecer ahorros energéticos, por ejemplo, redistribución de horarios o salones de acuerdo a la potencia instalada) y la organizacional (conformación e inclusión del comité de ahorro energético en los organismos que toman decisiones en la institución); igualmente, dichos factores junto con los otros resultados obtenidos del programa de gestión energética, deben ser entregados en un informe semestral y/o anual por el CAEMA a los directivos de la institución para su respectivo análisis. 


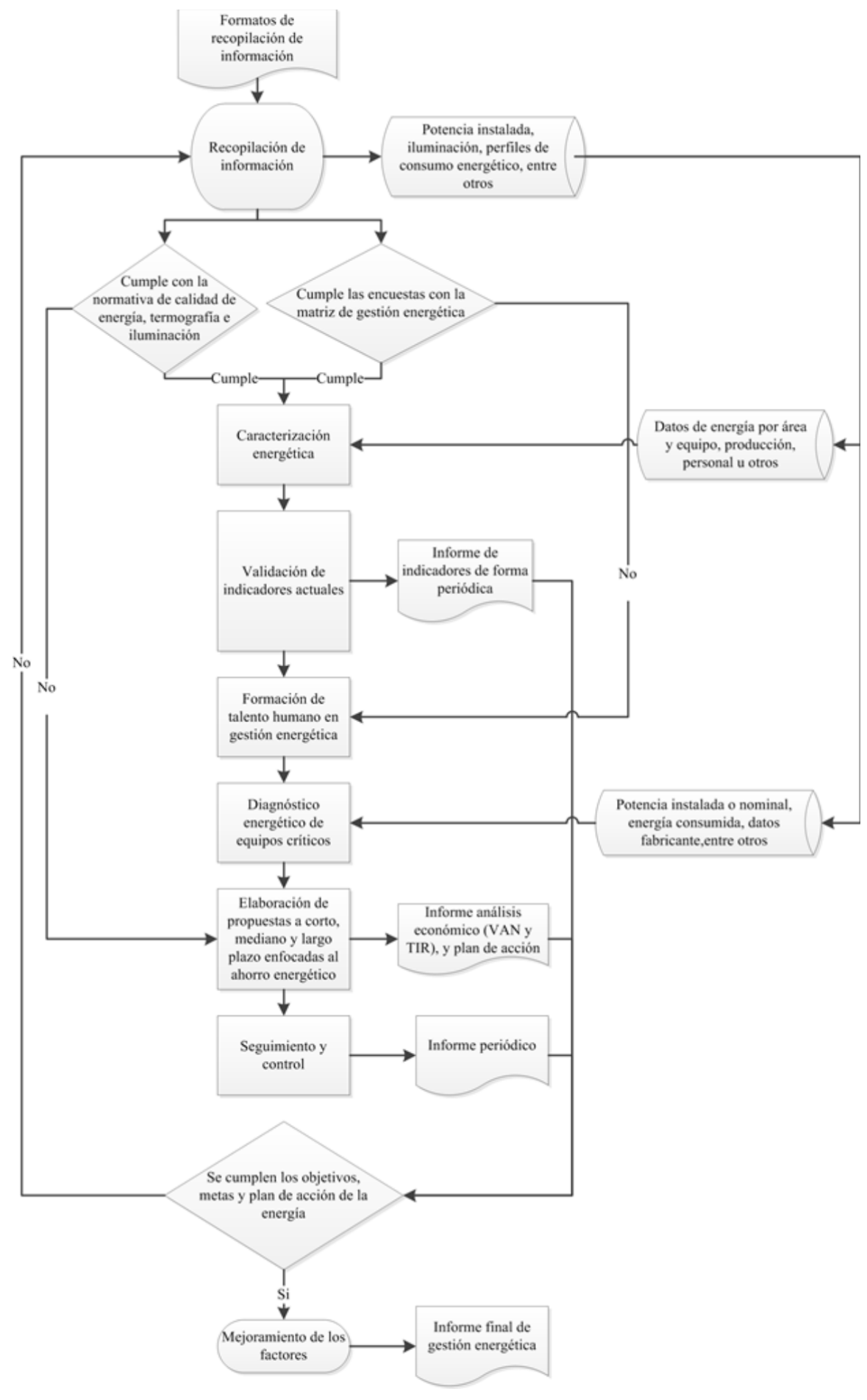

Fig. 3. Programa piloto de gestión energética propuesto para instituciones educativas en el departamento del Tolima 
3.3 Posible ahorro energético y de emisiones de $\mathrm{CO}_{2}$ con propuestas a corto, mediano y largo plazo en instituciones educativas oficiales en el departamento del Tolima

Al realizar la implementación de las propuestas a corto, mediano y largo plazo obtenidas en este estudio, se espera que el posible ahorro sea de 7025 MWh (30\%), equivalente a $\$ 2824$ millones, teniendo en cuenta un valor de $\$ 402 / \mathrm{kWh}$ para el mes de mayo del 2015. Además, el posible ahorro de emisiones de CO2 para el año 2014 al realizar la implementación de las propuestas a corto, mediano y largo plazo obtenidas en este estudio será de 1328,926 toneladas de $\mathrm{CO}_{2}$ (30\%). Adicionalmente, si los resultados obtenidos son satisfactorios, se implementaría en los 344 colegios privados restantes y posteriormente, a universidades.

Por otro lado, el presupuesto que se propone para implementar el programa piloto de gestión energética para instituciones educativas públicas en el departamento del Tolima es de aproximadamente \$4727 millones; asimismo, se requiere dividir el departamento en cuatro provincias (Centro, Norte, Oriente y Sur) para lograr abarcar todos los colegios y utilizar los equipos de medición de potencia por periodos de tiempo diferentes en cada una de ellas, puesto que permite disminuir a la mitad la cantidad de los equipos (24) para los 47 municipios del departamento del Tolima.
De igual modo, se recomienda iniciar en algunas provincias con la elaboración e implementación de los formatos para la recopilación de información y la caracterización de la institución educativa, mientras en las otras, se puede ir midiendo la capacidad instalada y el consumo energético en tiempo real con los analizadores de potencia, registradores de datos y pinzas amperimétricas digitales. Posteriormente, se realiza el cambio de actividades en las provincias.

3.4 Indicadores de gestión esperados para las instituciones educativas oficiales en el departamento del Tolima

Se calcularon los indicadores de gestión esperados a partir de la implementación del programa piloto de gestión energética en las instituciones educativas oficiales en el departamento del Tolima (ver Tabla 8), de acuerdo a: la cantidad de estudiantes, que son aproximadamente 327420 (Ministerio de Educación Nacional, 2012); 263 colegios públicos; y la energía eléctrica consumida con la disminución de 2410 $\mathrm{kWh} / \mathrm{mes}$, debido a las motobombas de la piscina y abastecimiento, puesto que normalmente no se tienen en un colegio público estándar.

Sin embargo, se debe aclarar que dependiendo del tipo de colegio (comercial o técnico), pueden presentarse mayores o menores valores de potencia instalada y/o consumos energéticos, por ejemplo, si se tiene taller de metal mecánica, se deben tener en cuenta los equipos de soldadura.

Tabla 8. Comparación de indicadores actuales y meta. Año 2014.

\begin{tabular}{|c|l|c|c|c|c|}
\hline Nivel & \multicolumn{1}{|c|}{ Indicador } & Unidad & $\begin{array}{c}\text { Valor promedio } \\
\text { esperado }\end{array}$ & $\begin{array}{c}\text { Valor meta } \\
\text { esperado }\end{array}$ & $\begin{array}{c}\text { \% de } \\
\text { disminución }\end{array}$ \\
\hline 3 & $\begin{array}{l}\text { Índice de consumo } \\
\text { energético por área total. }\end{array}$ & $\mathrm{kWh} / \mathrm{mes}-\mathrm{m}^{2}$ & 0,76 & 0,53 & $30 \%$ \\
\hline 3 & $\begin{array}{l}\text { Índice de consumo } \\
\text { energético por persona. }\end{array}$ & $\mathrm{kWh} /$ mes-persona & 5,93 & 4,14 & $30 \%$ \\
\hline 4 & Huella de Carbono & $\mathrm{KgCO}_{2} \mathrm{e} / \mathrm{mes}$ & 2100 & 1000 & $52 \%$ \\
\hline 4 & $\begin{array}{l}\text { Índice de emisiones de } \\
\mathrm{CO}_{2} \text { por área de edificio }\end{array}$ & $\mathrm{kgCO}_{2} /$ mes-m & 0,10 & 0,10 & $29 \%$ \\
\hline 4 & $\begin{array}{l}\text { Índice de emisiones de } \\
\mathrm{CO}_{2} \text { por persona }\end{array}$ & $\mathrm{kgCO}_{2} /$ mes-persona & 0,78 & 0,78 & $30 \%$ \\
\hline
\end{tabular}


De igual forma, al implementar el programa piloto, se espera poder comparar los valores obtenidos de los indicadores propuestos, mejorarlos y con ellos, lograr categorizar a los colegios en tres rangos: bueno, suficiente e insuficiente. Esto se haría con base en su eficiencia energética; posteriormente, se implementaría el programa piloto en colegios privados de la región.

También, se evidencia en la Tabla 8 que la mejora que se proyecta en tales indicadores oscila entre el 29 y el $52 \%$ y se espera obtener los siguientes indicadores de los niveles 3 y 4 propuestos: índice de consumo energético por área total de $0,53 \mathrm{kWh} / \mathrm{mes}-\mathrm{m}^{2}$; índice de consumo energético por persona, 8,18 kWh/mespersona; huella de carbono, $2100 \mathrm{~kg} \mathrm{CO} 2 \mathrm{e} / \mathrm{mes}$; índice de emisiones de por área de edificio, $0,10 \mathrm{~kg} \mathrm{CO} /$ mes$\mathrm{m}^{2}$ y el índice de emisiones de por persona, 0,78 kg cO2/mes-persona.

\section{CONCLUSIONES}

El colegio Champagnat no cuenta con políticas claras ni formación de talento humano en lo que respecta a realizar estrategias enfocadas al ahorro energético; adicional, las dependencias que intervienen en el sistema actual de control de costos no se reúnen en ningún tipo de comité para revisar el comportamiento energético de la institución educativa en términos administrativos y financieros. Además, las decisiones sobre el tema se toman por parte de la administradora y el rector y no de forma concertada con personal de otras áreas. Asimismo, se evidencia una oportunidad para que al conformar el Comité de Ahorro Energético, este flujo de información mejore y se logre crear en el personal de la institución un mayor sentido de pertenencia y una cultura energética y ambiental.

La caracterización energética permite en las instituciones educativas determinar: gráfico del proceso energético, perfiles de consumo de energía activa y reactiva, aforos o inventarios eléctricos de los equipos y/o máquinas, áreas y equipos que tienen mayor consumo energético. Así, a partir de los resultados obtenidos, se podrían realizar los diagnósticos y el análisis de calidad de energía con base en cálculos de ingeniería y tecnología y elaborar las propuestas a corto, mediano y largo plazo con o sin inversión económica enfocadas al ahorro energético. Esto permite replicar fácilmente el programa energético planteado en el presente trabajo al resto de instituciones educativas de secundaria a nivel internacional, nacional y en el departamento del Tolima.

Se establecieron indicadores energéticos aplicables a instituciones educativas, evaluándolos cuantitativamente, los cuales permiten comparar el nivel tecnológico de la instalación con la capacidad de potencia instalada de sus equipos y/o máquinas, los hábitos de las personas que viven o trabajan en ella y el régimen horario que involucra consumo de energía eléctrica. Por lo tanto, para poder controlar estos indicadores, es necesario tener medidores (contadores eléctricos o equipos especializados) ubicados estratégicamente en las zonas y procesos identificados que involucren cada uno de los indicadores planteados. De igual forma, la construcción de los indicadores no solo permite comparar internamente el nivel de eficiencia y mejoras que se van realizando, sino también elaborar la comparación con otras instituciones educativas a nivel departamental, nacional e internacional cuyas actividades estén relacionadas con la educación y tengan características constructivas similares.

De acuerdo con los resultados encontrados, se evidencia que la implementación de indicadores permite desarrollar el seguimiento de los consumos de energía y la gestión energética que se llevan a cabo en los procesos de las instituciones educativas; esto, teniendo en cuenta la norma ISO 50001 y los requerimientos para su aplicación, además, la posible aplicación de un programa de gestión de la energía, como lo establece en la norma, que involucre los indicadores propuestos y acciones correctivas tanto en la instalación como en los hábitos de los ocupantes. 
Se propuso un programa piloto de gestión energética para 263 instituciones educativas de secundaria oficiales en el departamento del Tolima; tiene los siguientes componentes: recopilación de información; caracterización energética; validación de los indicadores de gestión; formación de talento humano en gestión energética; diagnóstico energético de equipos críticos, propuestas a corto, mediano y largo plazo; seguimiento y control del programa.

Además, se espera que con este programa piloto, se logren mejorar los siguientes factores en los colegios: financiero, ambiental, social y operacional. Posteriormente, con los resultados obtenidos, se implementaría en los 344 colegios privados de la región.

AGRADECIMIENTOS: Al Colegio Champagnat de la ciudad de Ibagué y toda su comunidad académica, al permitir el ingreso a sus instalaciones y el acceso a la información requerida para llevar a cabo este estudio.

\section{REFERENCIAS}

Álvarez, S., Blanquer, M., \& Rubio, A. (2014). Carbon footprint using the Compound Method based on Financial Accounts. The case of the School of Forestry Engineering, Technical University of Madrid. Journal of Cleaner Production, 66, 224-232.

Arambula Lara, R., Pernigotto, G., Cappelletti, F., Romagnoni, P., \& Gasparella, A. (2015). Energy audit of schools by means of cluster analysis. Energy and Buildings, 95, 160-171.

Bunse, K., Vodicka, M., Schönsleben, P., Brülhart, M., \& Ernst, F. O. (2011). Integrating energy efficiency performance in production management e gap analysis between industrial needs and scientific literature. Journal of Cleaner Production, (19), 667679.

Campos Avella, J. C., Gómez Dorta, R., \& Santos Macías, L. (1997). La Eficiencia Energética en la Gestión Empresarial. Cien Fuegos, Cuba: Universidad de Cien Fuegos.

Dávila Collaguazo, F. J., \& Varela Rosario, D. S. (2014). Determinación de la huella de carbono en la Universidad Politécnica Salesiana, Sede Quito, Campus Sur. Quito: Universidad Politécnica Salesiana.

Dias Pereira, L., Raimondo, D., Paolo Corgnati, S., \& Gameiro da Silva, M. (2014). Energy consumption in schools - A review paper. Renewable and Sustainable Energy Reviews, 40, 911-922.

Dobes, V. (2013). New tool for promotion of energy management and cleaner production on no cure, no pay basis. Journal of Cleaner Production(39), 255-264. Economou, A. (2012). The use of natural gas and geothermal energy in school units. Greece: A case study. Renewable and Sustainable Energy Reviews, 16(2), 1317-1322. 
Gobierno Nacional. (2001). Ley 697. Bogotá.

Gobierno Nacional. (2003). Decreto 3683 de 2003. Recuperado el 11 de Junio de 2014 de http://www.alcaldiabogota.gov.co/sisjur/normas/No rma1.jsp?i=11032

Introna, V. C., Benedetti, M., \& Biagiotti, S. (2014). Energy Management Maturity Model: an organizational tool to foster the continuous reduction of energy consumption in companies. Journal of Cleaner Production, (83), 108-117.

Kim, T.-W., Lee, K.-G., \& Hong, W.-H. (2012). Energy consumption characteristics of the elementary schools in South Korea. Energy and Buildings, 54, 480489.

N. Larsen, H., Pettersen, J., Solli, C., \& G. Hertwich, E. (2013). Investigating the Carbon Footprint of a University - The case of NTNU. Journal of Cleaner Production, 48, 39-47.

Norma Técnica Colombiana (NTC). (10 de Junio de 2008). Norma NTC 5001. Recuperado el 31 de Enero de 2015, de

http://tienda.icontec.org/brief/NTC5001.pdf

Organización Internacional de Normalización (ISO). (Junio de 2011). Documento resumén ISO 50001. Recuperado el 4 de Octubre de 2013, de http://www.iso.org/iso/iso_50001_energy-es.pdf

Ozawa-Meida, L., Brockway, P., Letten, K., Davies, J., \& Fleming, P. (2013). Measuring carbon performance in a UK University through a consumption-based carbon footprint: De Montfort University case study. Journal of Cleaner Production, 56, 185-198.

Pinzón C., J. D., Corredor R., A., Santamaría P., F., Hernández M., J. A., \& Trujillo R., C. L. (2014). Implementación de indicadores energéticos en centros educativos. Caso de estudio: Edificio
Alejandro Suárez Copete-Universidad Distrital Francisco José de Caldas. Revista EAN, (77), 184-201.

San Miguel, P. A. (2009). Calidad. Madrid: Paraninfo S.A.

Skip Laitner, J. A. (2013). An overview of the energy efficiency potential. Environmental Innovation and Societal Transitions, (9), 38-42.

Torres E., D. (2002). Plan de eficiencia energética para una unidad educativa: Colegio Adventista ciudad de Quito (Tesis de pregrado). Quito: Universidad Internacional SEK.

Townsend, J., \& Barrett, J. (2013). Exploring the applications of carbon footprinting towards sustainability at a UK university: reporting and decision making. Journal of Cleaner Production, 1-13.

Unidad de Planeación Minero Energética (UPME) (Agosto de 2013). Programa Estratégico NacionalSistema de Gestión Integral de Energía. Recuperado el $21 \mathrm{de}$ jun io de $2014, \mathrm{de}$ http://www.grisec.unal.edu.co/Boletin4.pdf

Valencia Toro, Y. A., \& Vargas Londoño, P. A. (2013). La institución educativa El Retiro evalúa su huella de carbono en el corregimiento de Morelia, una apuesta desde la educación ambiental para la sostenibilidad. Pereira: Universidad Tecnológica de Pereira.

Yan, D., Zhe, T., Yong, W., \& Neng, Z. (2011). Achievements and suggestions of heat metering and energy efficiency retrofit for existing residential buildings in northern heating regions of China. Energy Policy, 39(10), 4675-4682.

Zapata Henao, J. L., \& González Franco, F. (2014). Uso eficiente y ahorro de energía eléctrica en el colegio Inem Felipe Pérez: Una visión estratégica desde la educación ambiental (Tesis de pregrado). Pereira: Universidad Tecnológica de Pereira. 